\title{
A 90 Minute Soccer Match Induces Eccentric Hamstring Muscles Fatigue
}

\author{
G. Kakavas ${ }^{1}$, N. Malliaropoulos ${ }^{2,3}$, T. Gabbett ${ }^{3,4}$, M. Mitrotasios ${ }^{4}$, N. Van Dyk ${ }^{5}$, G. \\ Bikos $^{8}$, N. Maffulli, $7,10,11$ \\ ${ }^{1}$ Fysiotek Spine and Sports Lab, Athens, Greece \\ 2 Sports and Exercise Medicine Clinic, Thessaloniki, Greece \\ ${ }^{3}$ Centre for Sports and Exercise Medicine, Queen Mary, University of London, London, UK \\ ${ }^{4}$ Gabbett Performance Solutions, Brisbane, Australia \\ ${ }^{5}$ Centre for Health Research, University of Southern Queensland, Ipswich, Australia \\ ${ }^{6}$ High School of Physical Education and Sports, National Kapodistrian University of Athens, Athens, Greece \\ 7 High Performance Unit, Irish Rugby Foorball Union, Dublin, Ireland \\ ${ }^{8}$ Euromedica Arogi Rehabilitation Clinic, Thessaloniki, Greece \\ ${ }^{9}$ Department of Musculoskeletal Disorders, School of Medicine and Surgery, University of Salerno, Salerno, Italy \\ ${ }^{10}$ Orthopedic Clinic, Ospedale San Giovanni di Dio e Ruggi D'Aragona, Salerno, Italy \\ ${ }^{11}$ School of Medicine, Keele University, Institute of Science and Technology in Medicine, Guy Hilton Research \\ Centre, Thornburrow Drive, Hartshill, Stoke-on-Trent, UK
}

\section{CORRESPONDING AUTHOR:}

Nicola Maffulli

Centre for Sports and Exercise Medicine

Queen Mary

University of London

Mile End Road

London, United Kingdom

E-mail: n.maffulli@qmul.ac.uk

DOI:

10.32098/mltj.02.2021.15

LEVEL OF EVIDENCE: 4

\section{SUMMARY}

Background/Purpose of the study. Hamstring injuries are the most prevalent muscle injuries in both amateur and professional soccer (football) players. Eccentric strength deficits and muscle strength imbalances have been associated with an increased risk of muscle injuries. The current study is a non-randomised observational study investigating whether fatigue significantly reduces peak eccentric strength of the hamstring muscle group.

Methods. Twenty-two right leg dominant professional football players participated in this study. None were injured or were rehabilitating from an injury at the time of testing. The eccentric strength of the hamstring muscles was determined using the Nordic exercise on a Norbord device prior to a 90 minute soccer match and after its end.

Results. There was a significant decrease in eccentric strength at the end of the match (before the match: $\mathrm{M}=306.91, \mathrm{SD}=59.26$; after the match: mean $=277.77 \pm \mathrm{SD}=$ $60.35 \mathrm{p}=.000$, Cohen's $\mathrm{d}=.50$ ). The imbalance in eccentric hamstring muscle strength between dominant and non-dominant limb before the match remained unchanged after its end.

Conclusions. A professional football match significantly impacts on the ability of players to produce high speed eccentric strength in the hamstring muscle groups. Resistance to fatigue and eccentric strength, particularly at high speeds, are considerable factors in conditioning of professional soccer players. Eccentric muscle strength fatigue of the hamstring muscle group after a 90 min soccer match may provide a possible explanation for the greater risk of hamstring injuries under fatigue conditions.

\section{KEY WORDS}

Adaptation; bamstring; injury prevention; risk factors scheduling. 


\section{BACKGROUND}

Participation in a single football match leads to acute fatigue, with a decline in physical performance over the following hours and days. In elite professional soccer, players can play up to 70 competitive matches per season. Hamstring injury (HI) is the most prevalent injury in both amateur (1) and professional (2) soccer (football) players, with an increase in the annual incidence of 2.4\% between 2010 and 2014 (3). Modifiable HSI risk factors such as eccentric knee flexor weakness (4) and muscle architecture, specifically biceps femoris fascicle length (4), are potential focus areas for $\mathrm{HI}$ injury prevention programs. A popular exercise choice for prevention of $\mathrm{HI}$ is the Nordic hamstring exercise (NHE), as it can influence both eccentric knee flexor strength and muscle architecture (4). Though ranked in the top five injury prevention modalities, compliance with the exercise is poor (5).

The adaptive response to eccentric strength training is multifactorial: it may include increases in motor unit discharge rate (6) and changes in muscle architecture, including hypertrophy and increase in fascicle length (7). The stimulus for hypertrophy is governed by mechanical tension and intramuscular metabolic stress (8), the degree of which may be influenced by the muscle status at either the beginning or end of football activity.

Neuromuscular fatigue has central and peripheral origins. Central fatigue, preponderant during long-duration low-intensity exercises, may involve a drop in the central command (motor, cortex, motoneurons) elicited by the activity of cerebral neurotransmitters and muscular afferent fibers. Peripheral fatigue, associated with an impairment of the mechanisms from excitation to muscle contraction, may be induced by a perturbation of the calcium ion movements, an accumulation of phosphate, and/or a decrease of the adenosine triphosphate stores (8).

The present study examined the changes in eccentric strength of the hamstring muscle group to ascertain whether the fatigue induced by a 90 minute match by elite professional soccer players influences two of the major risk factors for hamstring injury, namely 1) eccentric strength of the hamstring muscle group and 2) between limb imbalance in eccentric strength of the hamstring muscle group (9). Therefore, the current study tested the null hypothesis that a 90 minute match by elite professional soccer players did not produce any changes in the eccentric strength of the hamstring muscle group and did not result in changes in between limb imbalance in eccentric strength of the hamstring muscle group.

\section{MATERIALS AND METHODS}

Twenty-two professional football players (Mean \pm SD: Age: $19.3 \pm 2.9$ year; Height $185.0 \pm 8.7 \mathrm{~cm}$; Body Mass
$81.6 \pm 6.7 \mathrm{~kg})$ participated in this study. All players were right dominant (defined as their preferred kicking leg). Subjects were included in the study if they were not injured or rehabilitating from an injury at the time of testing. Ethics approval for the study was granted by our University. Written informed consent was obtained from each player or their legal guardian prior to data collection. Participants were blinded to the study hypothesis. The study was carried out in accordance with the ethical standards of the journal (10) and with the Declaration of Helsinki, and was approved by our local Ethics Committee.

The game was played in the second half of the competition season. The eccentric hamstring strength of players was assessed using the NORDBORD (Vald Performance, Queensland, Australia) device prior to the 90 minute soccer match and immediately after it. Players were examined on the field before performing a $90 \mathrm{~min}$ football match. The Nordic board test was used to measure hamstring strength based on the NHE. We used 2 NORDBORDS per each team, and each athlete undertook a NORDIC exercise on the NORDBORD. The 11 players in each team completed the testing procedure within $20 \mathrm{~min}$ before and after the match.

\section{Data analysis}

The Shapiro-Wilk test confirmed that the data followed the normal distribution. We tested whether the eccentric force of the right and left hamstring muscle group in each player changed before and after the match, using a paired sample t-test and one sample t-test. Cohen's d index was used to estimate the intensity of the difference whenever that was necessary. The statistical analysis was conducted by using the statistical software SPSS 23.0.

\section{RESULTS}

There was a significant difference in the eccentric strength of the hamstring muscle group before a match $(\mathrm{M}=306.91$, $\mathrm{SD}=59.26)$ compared to after the match (mean $=277.77$ $\pm \mathrm{SD}=60.35 \mathrm{p}=.000$, Cohen's $\mathrm{d}=.50)$. The $95 \%$ confidence interval $(24.00,34.68)$ showed a decrease of $7.82 \%$ to $11.30 \%$ from the pre-match eccentric force $(M=306.91)$ of the athletes (figure 1). There was no statistical difference between the mean value of hamstring imbalance between the right and left hamstring $(\mathrm{M}=1.49, \mathrm{SD}=8.96)$ and zero value; $\mathrm{t}(21)=.777, \mathrm{p}=.446$. Also, the bootstrap $95 \%$ confidence interval $(-2.18,5.11)$ suggest that there is no hamstring imbalance (figure 2 ). 


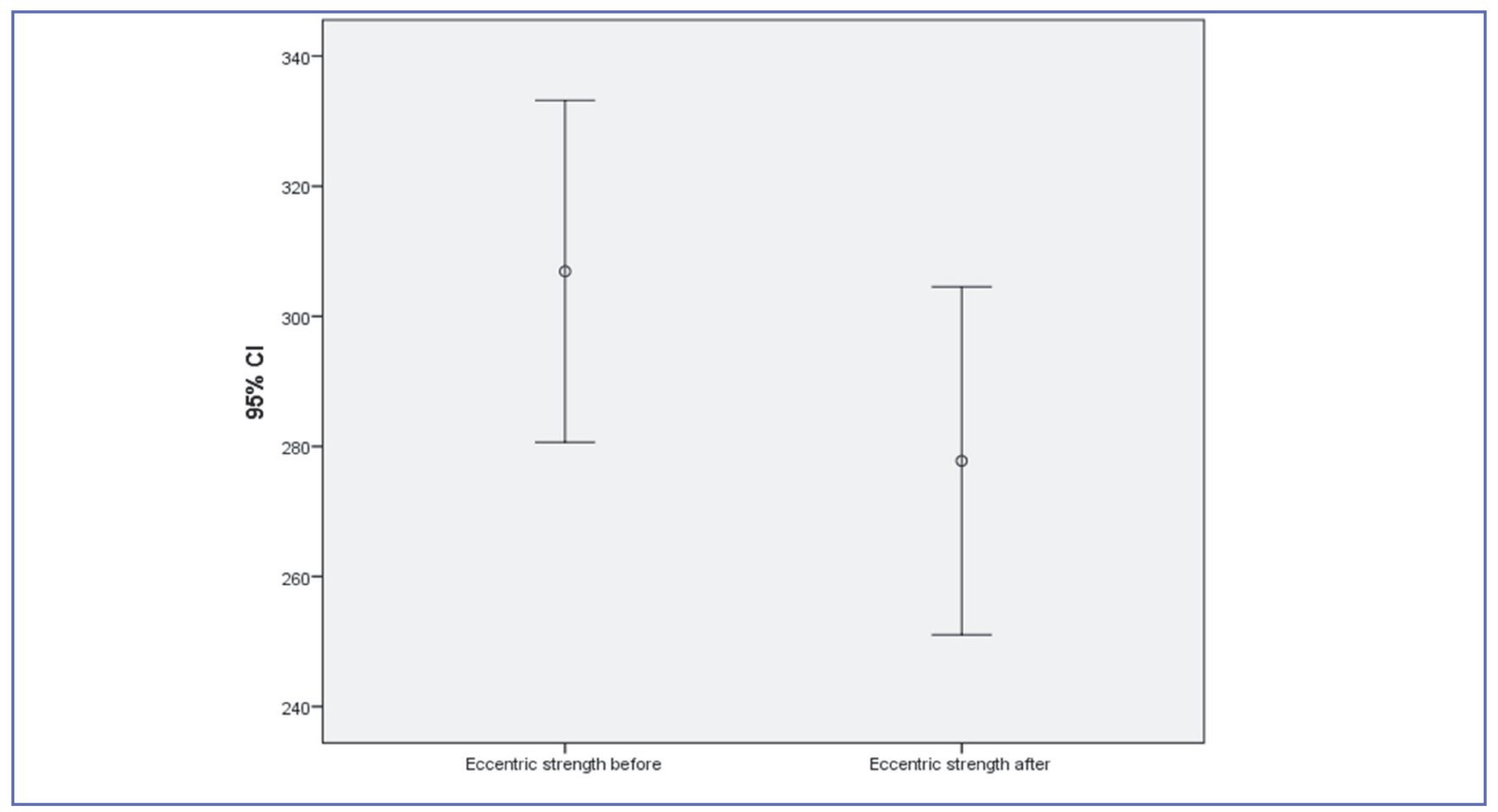

Figure 1. Eccentric strength before and after the exercise, $95 \%$ confidence intervals.

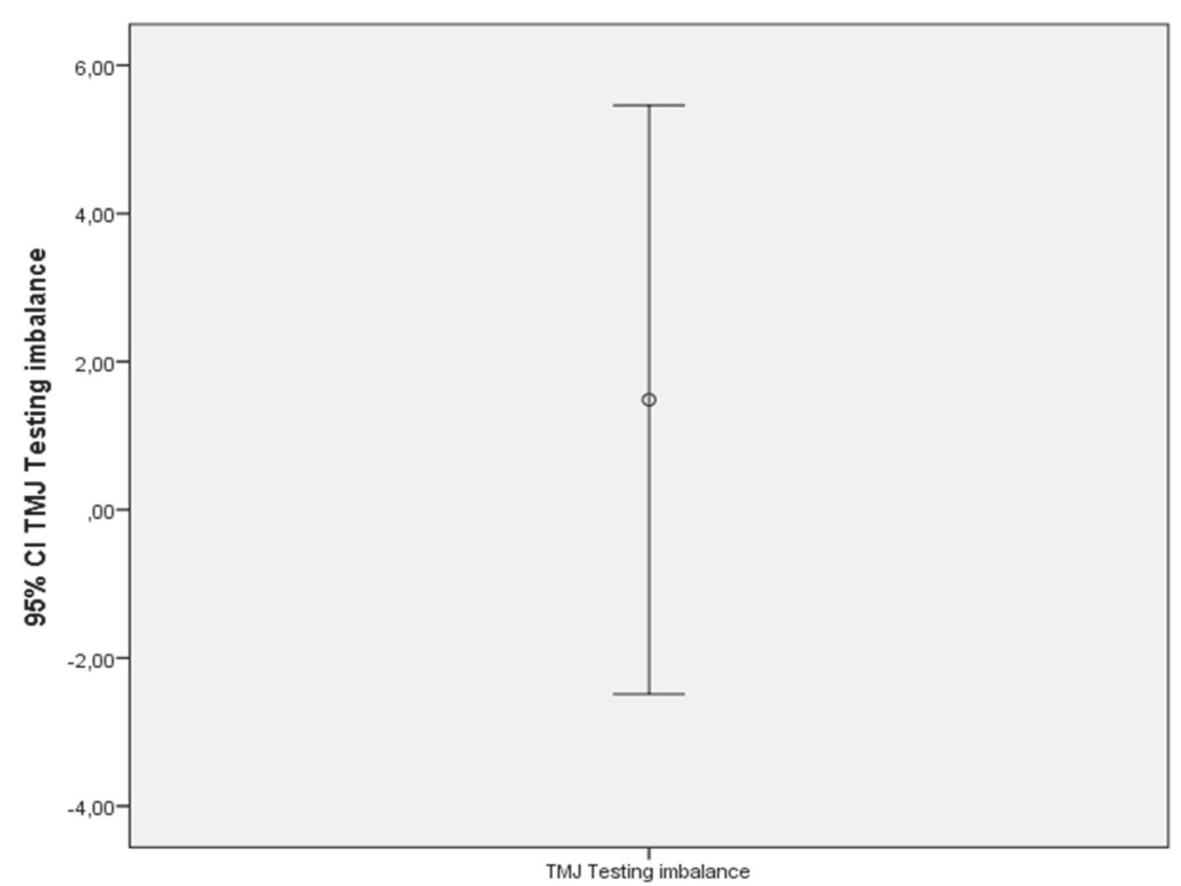

Figure 2. Hamstring testing imbalance (after the exercise), 95\% confidence intervals. 


\section{DISCUSSION}

Soccer involves sprinting, changes in direction and running speed, jumps and tackles, as well as technical actions such as dribbling, shooting and passing. Fatigue may induce a decline in performance. In soccer, fatigue occurs temporarily after short-term intense periods in both halves, towards the end of the match, and persists after the match. Rampinini et al., for example, observed reductions in knee extensor maximal voluntary activation and electromyographic activity and a decrease in knee extensor peak torque responses to paired stimulations after a match (11).

Match-related fatigue results from a combination of central and peripheral factors, involving mechanisms from the central nervous system to the muscle cell itself and energy production. The present investigation showed a significant decrease in eccentric strength of both right and left hamstring muscle groups after the game, with no change in muscle imbalance detected between the left and right hamstring muscle groups. Factors associated to an increased risk of muscular strain injury include poor muscle strength, particularly eccentric strength deficits, and ipsilateral muscle strength imbalances (12). The temporal pattern of injury during match play also suggests that fatigue is a factor in injury causation, with $47 \%$ of match-play hamstring strains incurred during the latter stages of each half (11). Soccer is characterised by an irregular and intermittent activity profile; this intermittent nature places great emphasis on acceleration and deceleration phases of sprinting (13).

Fatigue has become a hot topic in football, and is regarded as a complex and multi-factorial phenomenon (11). There is growing interest as to how fatigue relates to recovery, player fitness and effort during matches. To avoid exhaustion before the final whistle, players may adopt a pacing strategy that allows them to be involved in demanding and critical moments, even during the final stages of a match (14). The regulation of self-chosen high intensity activity is also an important product of training, as players need to learn to adopt pacing strategies that will allow a high effort even during the final stages, and whenever required of the match situation (15). Fatigue during a soccer match has been associated with decreased eccentric hamstring strength, and this may be related to increased hamstring injury risk: nearly half of all hamstring injuries occur during the final $15 \mathrm{~min}$ of each half. Therefore, it could be hypothesized that fatigue during the later stages of a soccer match may increase the predisposition to hamstring strain injury by negatively altering the biomechanics of sprinting in relation to muscle flexibility, muscle strength, or body mechanics (16).

There is a significant decrease in eccentric hamstring strength during simulated soccer match-play (17). A complex neuro- muscular coordination pattern is necessary during the running cycle (18). Therefore, if the hamstrings are not able to produce sufficient strength to decelerate the limb during the latter part of the swing phase, eccentric overload could cause tears at the musculotendinous unit (19). Hamstring injuries are most frequently sustained at the end of matches and training sessions (11). This would support the notion that fatigue may be a predisposing factor for such injuries. Any factor that adversely affects the appropriate running pattern may result in injury. The dual innervation of the biceps femoris may cause asynchrony, as poor coordination may allow separate parts of the muscle to activate at different times. The observed decline in eccentric hamstrings strength might be attributed to the greater contribution of the hamstrings in controlling the intermittent running profile (2). Additionally, as sprinting is a primary mechanism of injury, the frequency of speed changes places greater emphasis on the acceleration and deceleration phases of the running cycle (20). The increased muscle contribution from the biceps femoris in maintaining running mechanics during the intermittent protocol, in parallel with the decrease in peak eccentric strength, may further increase the risk of injury.

The test-retest reliability of the NORBORD device (4) is high to moderate when NHE measurements were performed bilaterally, but there is poor reliability during unilateral testing. Also, elite athletes with a unilateral history of hamstring injury within the previous 12 months displayed significant eccentric knee flexor weakness in their injured limb and to the limbs of uninjured recreational athletes (21). In our setting, the results obtained were valid and reliable.

Most biceps femoris injuries occur at the end of matches and training sessions (2). Moreover, when soccer players become fatigued during maximal sprinting (22), early activation of the biceps femoris and semitendinosus muscles occurs during the swing phase of the cycle (23). This recruitment pattern may result from local muscle fatigue, and may be causative of hamstring strains (24). In agreement, as fatigue increases, so does biceps femoris activity during knee extension (concentric quadriceps contraction) movements in recreational soccer players (24).

All these studies highlight a possible positive relationship between fatigue and hamstring strains. Despite this plausible link, however, to our knowledge no study examines these variables in elite athletes. However, in vitro animal studies further support such hypothesis (15). The extensor digitorum longus muscles from 48 rabbits were fatigued to different levels of severity, then stretched to failure, and compared with their non-fatigued contralateral controls. Fatigued muscles are able to absorb less energy before reaching the degree of stretch that causes injury, suggest- 
ing that fatigue is an important factor in the pathogenesis of acute muscle strains (23). However, it should also be noted that muscles were injured at the same length, regardless of fatigue. Fatigued player may become more susceptible to both muscular strain injury and impaired joint stability (22). Coaching and medical staff need to ensure that players are sufficiently warmed up before the start of the second half of the match, and that their eccentric hamstrings strength is well developed and resistant to fatigue.

\section{Limitations}

We are aware that this investigation included a relatively small number of participants, and this would limit the generalizability of our findings. However, we point out that our study included only elite professional players who were extremely well trained, and that the index match occurred in mid-season.

\section{CONCLUSIONS}

Soccer specific fatigue can influence the eccentric strength of the hamstring muscle group, and thus exert an influence of the propensity of football players to injury to these muscles. However, it does not exert any influence on the imbalance of the hamstrings between the dominant and non-dominant leg. These findings highlight not only the susceptibility of eccentric hamstrings strength to fatigue but also the influence imposed by movement speed. The present study confirms previous findings (25) but was performed in real match play conditions, and therefore is even more specific and valid.

Strength work is a fundamental component of the conditioning program in professional soccer. However, strength and speed training tend to be addressed only when the players are well rested. Such practices may not provide the best mode of intervention to prevent the increased injury incidence observed late in the game. Resistance to fatigue and eccentric strength, particularly at high speeds, should be given greater consideration in conditioning for soccer.

\section{Practical implications}

There was a significant decrease in eccentric strength of both hamstrings before and to after the game.

\section{REFERENCES}

1. van Beijsterveldt AM, van de Port IG, Vereijken AJ, Backx FJ. Risk factors for hamstring injuries in male soccer players: a systematic review of prospective studies. Scand J Med Sci Sports 2013;23:253-62.
There was no effect on muscle imbalance between the dominant and non-dominant hamstring muscle group.

Coaches, fitness coaches, rehabilitation specialists and sports medicine doctors should be made aware that a single match impacts negatively on the ability of football players to develop eccentric strength in the hamstring muscle group, so that they can plan training and prevention programmes adapted to these findings.

Soccer specific fatigue affects the eccentric strength of the hamstring muscle group.

There is significant decrease in eccentric strength of both hamstring muscle group after a match.

Future injury prevention strategies may need to consider reducing negative effects of fatigue.

\section{CONTRIBUTIONS}

All authors contributed to the study conception and design. Material preparation, data collection and analysis were performed by Georgios Kakavas. The first draft of the manuscript was written by Georgios Kakavas and Tim Gabbett, Michail Mitrotasios, Nicol Van Dyk, Nikolaos Malliaropoulos, Georgios Bikos and Nicola Maffulli participated to review and editing of the manuscript. All authors read and approved the final manuscript.

\section{RIGHTS}

All procedures performed in the current study involving human participants were in accordance with the ethical standards of the Atromitos Fc Ethics Committee (reference number 1023) and with the 1964 Helsinki declaration and its later amendments.

\section{INFORMED CONSENT}

Written informed consent was obtained from each player or their legal guardian prior to data collection.

\section{CONFLICT OF INTERESTS}

The authors declare that they have no conflict of interests.

2. Woods C, Hawkins RD, Maltby S, Hulse M, Thomas A, Hodson A. The Football Association Medical Research Programme: an audit of injuries in professional football--analysis of hamstring injuries. Br J Sports Med 2004;38:36-41. 
3. Ekstrand J, Walden M, Hagglund M. Hamstring injuries have increased by $4 \%$ annually in men's professional football, since 2001: a 13-year longitudinal analysis of the UEFA Elite Club injury study. Br J Sports Med 2016;50:731-7.

4. Timmins RG, Bourne MN, Shield AJ, Williams MD, Lorenzen C, Opar DA. Short biceps femoris fascicles and eccentric knee flexor weakness increase the risk of hamstring injury in elite football (soccer): a prospective cohort study. Br J Sports Med 2016;50:1524-35.

5. McCall A, Carling C, Nedelec M, et al. Risk factors, testing and preventative strategies for non-contact injuries in professional football: current perceptions and practices of 44 teams from various premier leagues. Br J Sports Med 2014;48:1352-7.

6. Higbie EJ, Cureton KJ, Warren $3^{\text {rd }}$ GL, Prior BM. Effects of concentric and eccentric training on muscle strength, cross-sectional area, and neural activation. J Appl Physiol (1985) 1996;81:2173-81.

7. Franchi MV, Atherton PJ, Reeves ND et al. Architectural, functional and molecular responses to concentric and eccentric loading in human skeletal muscle. Acta Physiol (Oxf) 2014;210:642-54.

8. Douglas J, Pearson S, Ross A, McGuigan M. Chronic Adaptations to Eccentric Training: A Systematic Review. Sports Med 2017;47:917-41.

9. Freckleton G, Pizzari T. Risk factors for hamstring muscle strain injury in sport: a systematic review and meta-analysis. $\mathrm{Br}$ J Sports Med 2013;47:351-8.

10. Padulo J, Oliva F, Frizziero A, Maffulli N. Muscles, Ligaments and Tendons Journal - Basic principles and recommendations in clinical and field Science Research: 2018 update. Muscles Ligaments Tendons J 2018;8(3):305-7.

11. Rampinini E, Bosio A, Ferraresi I, Petruolo A, Morelli A, Sassi A. Match-related fatigue in soccer players. Med Sci Sports Exerc 2011;43:2161-70.

12. Croisier JL, Forthomme B, Namurois MH, Vanderthommen M, Crielaard JM. Hamstring muscle strain recurrence and strength performance disorders. Am J Sports Med 2002;30:199-203.

13. Mjolsnes R, Arnason A, Osthagen T, Raastad T, Bahr R. A 10 -week randomized trial comparing eccentric vs. concentric hamstring strength training in well-trained soccer players. Scand J Med Sci Sports 2004;14:311-7.
14. Banister EW, Calvert TW, Savage MV, T B. A systems model of training for athletic performance. Austr J Sports Med Exerc Sci 1975;7:57-61.

15. Mair SD, Seaber AV, Glisson RR, Garrett WE, Jr. The role of fatigue in susceptibility to acute muscle strain injury. Am J Sports Med 1996;24:137-43.

16. Gabbett TJ, Hulin BT, Blanch P, Whiteley R. High training workloads alone do not cause sports injuries: how you get there is the real issue. In: Br J Sports Med 2016;50:444-5.

17. Orchard J, Best TM. The management of muscle strain injuries: an early return versus the risk of recurrence. Clin J Sport Med 2002;12:3-5.

18. Rogalski B, Dawson B, Heasman J, Gabbett TJ. Training and game loads and injury risk in elite Australian footballers. J Sci Med Sport 2013;16:499-503.

19. Foster C, Snyder A, Welsh R. Monitoring of Training, Warm Up, and Performance in Athletes. In: Lehmann M, Foster C, Gastmann U, Keizer H, Steinacker JM (editors), Overload, Performance Incompetence and Regeneration in Sport, 1st ed. Boston, Springer 1999;43-51.

20. Murray NB, Gabbett TJ, Townshend AD, Blanch P. Calculating acute: chronic workload ratios using exponentially weighted moving averages provides a more sensitive indicator of injury likelihood than rolling averages. Br J Sports Med 2017;51:749-54.

21. Opar DA, Piatkowski T, Williams MD, Shield AJ. A novel device using the Nordic hamstring exercise to assess eccentric knee flexor strength: a reliability and retrospective injury study. J Orthop Sports Phys Ther 2013;43:636-40.

22. Pinniger GJ, Steele JR, Groeller H. Does fatigue induced by repeated dynamic efforts affect hamstring muscle function? Med Sci Sports Exerc 2000;32:647-53.

23. Heiderscheit BC, Hoerth DM, Chumanov ES, Swanson SC, Thelen BJ, Thelen DG. Identifying the time of occurrence of a hamstring strain injury during treadmill running: a case study. Clin Biomech (Bristol, Avon) 2005;20:1072-78.

24. Wright J, Ball N, Wood N. Fatigue, H/Q ratios and muscle coactivation in recreational football players. Isokinet Exerc Sci 2010;17:161-7.

25. Small K, McNaughton L, Greig M, Lovell R. The effects of multidirectional soccer-specific fatigue on markers of hamstring injury risk. J Sci Med Sport 2010;13:120-5. 